\title{
Study Site Investigator
}

National Cancer Institute

\section{Source}

National Cancer Institute. Study Site Investigator. NCI Thesaurus. Code C93404.

A researcher at a study site who oversees multiple aspects of the study at a site, including protocol submission for IRB approval, participant recruitment, informed consent, data collection, and analysis. 